tectaron anticuerpos contra el medicamento. Los resultados de laboratorio no mostraron alteraciones en los signos vitales o electrocardiográficos que afectaran a los pacientes.

En conclusión, el liraglutide permitió reducir las concentraciones de glucosa en sangre en pacientes con diabetes tipo 2 sin provocar el aumento de peso o del riesgo de hipoglucemia. (Madsbad S, Schmitz O, Ranstam J, Jakobsen G, Matthews DR, et al. Improved glycemic control with no weight increase in patients with type 2 diabetes after oncedaily treatment with the long-acting glucagon-like peptide 1 analog liraglutide (NN2211). A 12-week, double-blind, randomized, controlled trial. Diabetes Care. 2004;27:1335-42.)

\section{Algoritmos para determinar el umbral de alerta ante epidemias de malaria}

Los sistemas sanitarios requieren de mecanismos que les permitan pronosticar el incremento inusual del número de casos de determinadas enfermedades. Esos sistemas son particularmente necesarios en regiones propensas a la aparición de epidemias, ya que permiten emprender acciones oportunas de control y mitigar el impacto de esas epidemias sobre la salud de la población.

Por lo general, la detección de un número inusual de casos de una enfermedad en un momento determinado puede ser un indicio de que una epidemia está por aparecer. Los sistemas de alerta epidemiológica se basan en algoritmos diseñados para calcular los valores umbrales (número de casos) que pueden indicar con mayor certeza y antelación la aparición de las epidemias.

Sin embargo, hasta el momento no se han desarrollado métodos que puedan evaluar los sistemas de detección temprana y su capacidad para generar alertas oportunas. La utilidad de estos sistemas de alerta depende de la sensibilidad y la especificidad con que permitan identificar las epidemias en sus fases más tempranas.

En el caso de la malaria, se ha demostrado la utilidad de realizar intervenciones oportunas - como la eliminación de larvas, la fumigación de los residuos domésticos y la administración masiva de medicamentos- para controlar las epidemias, pero no se conoce aún la forma de identificar el momento óptimo para emprender esas acciones, especialmente cuando los recursos son escasos.

En este trabajo se describe un método empleado para evaluar varios sistemas sencillos de detección temprana de epidemias de malaria, según su capacidad para brindar alertas sensibles, específicas y oportunas. Para ello se emplearon los datos semanales de casos confirmados de malaria en 10 localidades de Etiopía entre 1999 y 2000. Los datos se obtuvieron según las semanas etíopes (que pueden ser de 5 a 9 días), por lo que se normalizaron para el análisis.

Se analizaron cuatro algoritmos para el cálculo del umbral de alerta. En todos los casos, la alerta se establecía si el número semanal de casos excedía ese umbral durante dos semanas consecutivas, a fin de aumentar la especificidad del sistema de alerta, independientemente del algoritmo empleado.

Los algoritmos analizados se basaron en los percentiles semanales (según el número de casos informados en esa misma semana durante los años estudiados, excepto en el año en cuestión); la media semanal más su desviación estándar (la media semanal del número de casos más un número dado de desviaciones estándar); el porcentaje de muestras positivas (proporción de muestras positivas con relación al número de muestras analizadas); y la pendiente del número de casos positivos semanales en una escala logarítmica (el umbral se define según la pendiente del logaritmo natural del número de casos semanales normalizados).

Cada algoritmo se evaluó según el número de alertas que hubiera desencadenado y el número de casos que se hubieran podido prevenir (NCP) si se hubiera aplicado la alerta. El NCP se definió como una función del número de casos detectados entre la $2 .^{a}$ y la $8 .^{a}$ semanas posteriores a la alerta, o entre la 2. ${ }^{a}$ y la $24 .^{a}$ semanas, en dependencia de la duración de las medidas de intervención.

En el período evaluado se detectaron 687903 casos de malaria. En promedio, cada una de las 10 instalaciones sanitarias participantes (una por cada distrito) trató entre 11 y 19 casos de malaria diariamente y más de 300 casos diarios durante las temporadas de mayor transmisión.

Según el número de alertas desencadenadas y la proporción de NCP para cada umbral, los sistemas de alerta basados en los algoritmos estudiados hubieran podido prevenir un mayor número de casos que las alertas anuales aleatorias.

El umbral de alerta basado en los percentiles logró resultados similares o mejores que los otros algoritmos según el número de alertas que hubiera desencadenado. Para ese número de alertas, este algoritmo hubiera prevenido la mayor proporción de casos en comparación con los otros métodos. Con relación al momento óptimo de declarar la alerta, el algoritmo basado en los percentiles también tuvo el mejor rendimiento. El algoritmo basado en la pendiente del número de casos en la escala logarítmica ofreció resultados ligeramente mejores que el sistema de alertas aleatorias, pero fue el peor de los algoritmos estudiados. El algoritmo basado en la media de los casos semanales más su desviación es- 
tándar tuvo su mejor desempeño cuando se emplearon los datos normalizados.

Cuando se emplearon umbrales de mayor especificidad (a manera de desencadenar el menor número de alertas), los métodos basados en la pendiente logarítmica y en el porcentaje de muestras positivas generaron la menor proporción de NCP en comparación con el resto de los algoritmos. Cuando se usaron umbrales de mayor sensibilidad se obtuvieron los resultados opuestos. En este caso, el algoritmo basado en el porcentaje de muestras positivas con un umbral $\leq 65 \%$ ofreció la mayor proporción de NCP en comparación con los otros algoritmos.

El empleo de una alerta anual con una intervención en una misma semana del año (por lo general inmediatamente antes del comienzo de la temporada de mayor transmisión) hubiera logrado prevenir $28,4 \%$ del NCP. Sin embargo, los algoritmos basados en la media semanal y los percentiles hubieran prevenido la misma proporción de casos con la mitad de las alertas anuales.

Si se hubiera empleado el percentil 85 como umbral para declarar las alertas, se habrían declarado 0,72 alertas anuales y se habría evitado $31,9 \%$ del NCP, mientras que la aplicación de umbrales basados en los percentiles 80 y 75 habría desencadenado 0,79 y 0,9 alertas anuales con $32,6 \%$ y $31,2 \%$ de NCP, respectivamente. El incremento del número de alertas anuales en $0,1-0,2$ no habría generado beneficios adicionales. En situaciones en la que el costo no constituye un factor limitante y es posible aplicar medidas preventivas anuales, el método basado en la proporción de muestras positivas puede ser el método de elección. Este método dio resultados similares o mejores que los otros métodos cuando se modelaron para realizar una alerta anual, ya que permitió identificar mejor el momento oportuno para la intervención.

Este estudio demuestra que con el empleo del percentil semanal de casos para determinar el punto de corte que define las situaciones de alerta de epidemias de malaria se pueden identificar tempranamente los períodos de mayor número de casos de esta enfermedad en Etiopía. Esto permitiría realizar intervenciones más racionales para el control de la malaria y evitaría un mayor número de casos de malaria.

La técnica comparativa expuesta en este trabajo puede emplearse para evaluar otros sistemas de alertas en otras poblaciones y con otras enfermedades. (Teklehaimanot HD, Schwartz J, Teklehaimanot A, Lipsitch M. Alert threshold algorithms and malaria epidemic detection. Emerg Infect Dis [serial on the Internet]. 2004;10(7). Hallado en: http://www.cdc.gov/ncidod/EID/vol10no7/030722.htm. Acceso el 29 de junio de 2004.)

\section{Síndrome de cansancio crónico y discapacidad laboral}

Según los Centros para el Control y la Prevención de Enfermedades (CDC) de los Estados Unidos de América, el síndrome de cansancio crónico (SCC) se define como un cansancio intenso que incapacita a los pacientes tanto física como mentalmente durante seis meses o más, que se exacerba por esfuerzos mínimos y que no se puede explicar mediante diagnósticos convencionales. Aunque en la actualidad existen varias definiciones operacionales diferentes del SCC, todas se basan en la información que aportan los pacientes. Debido a que no se cuenta con marcadores diagnósticos de laboratorio ni se han definido muestras de biopsia patognomónicas, el diagnóstico de esta enfermedad se realiza por exclusión. El SCC es una enfermedad puramente subjetiva y hasta el momento no se ha informado de tratamientos que hayan logrado mitigar la enfermedad, aunque la psicoterapia conductual cognoscitiva puede aliviar los síntomas.

El objetivo de este trabajo fue evaluar la información científica publicada sobre la detección y el control de la discapacidad en personas con SCC. Para ello se realizó una revisión sistemática en la que participó un panel multidisciplinario de expertos que evaluó los artículos publicados entre el $1 .^{\circ}$ de enero de 1988 (año en que se publicó la primera definición operacional de esta enfermedad) y el 15 de noviembre de 2001. La búsqueda bibliográfica se realizó en las bases MEDLINE, Current Contents, Cochrane Library y PsychINFO. Además se buscaron citas relacionadas con el tema en los estudios y artículos de revisión aceptados entre el $1 .^{\circ}$ de enero de 1999 y el 31 de diciembre de 2001.

Se tomaron en cuenta los estudios observacionales (prospectivos, retrospectivos y transversales) e intervensionistas, que informaran el diagnóstico de SCC según alguna de las cuatro definiciones para adultos de los CDC y que contuvieran los términos "fatigue syndrome" + "chronic" o "chronic fatigue". Solo se tomaron en cuenta los artículos publicados en inglés.

En total se identificaron 3640 estudios, de los cuales 53 cumplieron con los criterios de selección. De los estudios aceptados, 36 eran observacionales (3 210 pacientes) y en 17 se habían aplicado diferentes intervenciones (1 348 pacientes). Todos los 17 informaron de haber obtenido mejoras durante el seguimiento. Las intervenciones aplicadas fueron conductuales (cuatro estudios), psicológicas (dos estudios), con medicamentos (cinco estudios), ocupacionales (dos estudios) o combinadas (cuatro estudios). Seis estudios tenían grupos con placebo.

En total, 37 estudios informaron de la condición laboral de los pacientes y realizaron alguna me- 